\title{
REFERENCIAS A FERNÁNDEZ DE SANTAELLA EN EL LEXICON ECCLESIASTICUM DE DIEGO JIMÉNEZ ARIAS
}

\author{
Antonia M. ${ }^{a}$ Medina Guerra \\ Universidad de Málaga
}

\begin{abstract}
El Lexicon ecclesiasticum latino-hispanicum de Diego Jiménez Arias se imprime por primera vez en Salamanca («In ædibus Andreæ a Portonariis»), en 1566. En las siguientes salidas se hicieron multitud de adiciones y correcciones que mantuvieron viva la obra hasta bien entrado el siglo XVIII, aunque no siempre significaron mejoras de la obra original. Así, lo advierte uno de los adicionadores del Lexicon, Martín David ${ }^{1}$, en las palabras que dedica «Al lector»:
\end{abstract}

La primera edición de efte Vocabulario es de Roderico Ferdinando de Santa Ella, año de 1550. y aún dejpués en otra le añadió, y corrigió él mi Jmo en el de 1562 . Y en el de $1565^{2}$. el M. R. P. Fr. Diego Ximénez Arias, de la Sagrada Orden de Predicadores, hizo de él nueva dijpojición, y forma, y le facó con título de Lexicon Eccle Jia Jticum, enriqueciéndole de muchas voces, con autoridades de la Sagrada Ejcritura, comentos de muchos lugares de ella, y teftimonios de Santos Padres, Concilios, Decretos \&c. con mucha más difufión, y energía que Roderico, y enmendando a éfte muchas interpretaciones viciojas, que corrían en ju Vocabularium Eccle Jia Jticum [...]. Sobre ejta edición del P. Ximénez de Arias, en el

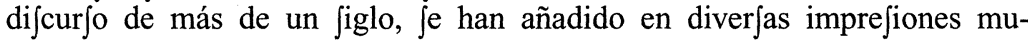
chas voces, y jignificados, con difuJas noticias Jagradas y humanas, por lo

\footnotetext{
${ }^{1}$ La primera edición en la que aparecen las correcciones y adiciones de Martín David es la impresa por Jacobo de Bordazar en Valencia en 1685 (véase Joaquín Hazañas y la Rúa, Maese Rodrigo (1444-1509), Sevilla, Librería e Imp. de Izquierdo y Comp. ., 1909, pág. 42).

2 Todos los catálogos que he tenido ocasión de consultar recogen la edición salmantina de 1566 como la primera corregida y adicionada por Diego Jiménez Arias. Se trata, sin duda, de una confusión, pues Martín David yerra también al darnos como primera edición del Vocabularium ecclesiasticum la de 1550 (fue publicado en Sevilla en 1499) al afirmar que su autor lo corrigió años más tarde en 1562 (Rodrigo Fernández de Santaella había muerto en la capital hispalense el 20 de enero de 1509).
} 


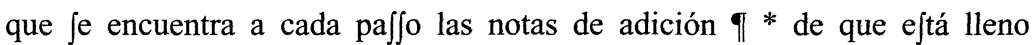
el Vocabulario: a cauja de que en las más imprefiones fe procura añadir, por jolicitar ejte título frequentemente (ajsí en éfte, como en otros Libros) quien procura lograr por ejta novedad mayor crédito en ju imprejión. Y a $\int i ́$, advirtiendo que en aquellas más je atendió a añadir dicciones, que a reconocerlas; y más a bujcar voces, que a perficionarlas: y que aunque algunas je han reducido a lo corriente (ejto je dice más en particular del Cajtellano) Jobre jer pocas, o han quedado con el trage de dejcompuejta Ortographía, o con el mal jonido de la antigua pronunciación: Y ajsimijmo, que muchas voces que eftaban en éfte, y en otros Vocabularios y Autores antiguos, es forzo jo emmendarlas, y perficionarlas, reduciéndolas a lo más corriente de efte tiempo $[\ldots]^{3}$.

A Martín David ${ }^{4}$ no se le oculta, pues, que el verdadero autor de la obra que corregía era Rodrigo Fernández de Santaella por más que en la portada siga conservando el nombre de Diego Jiménez Arias.

Pese a que en ediciones posteriores se pone de manifiesto que el Lexicon ecclesiasticum es una corrección y ampliación del Vocabularium ecclesiasticum de Rodrigo Fernández de Santaella, Jiménez Arias pretende, como es lógico, demostrar el aporte fundamental y revolucionario que supone su diccionario en el estudio de la lengua latina. El objetivo de su obra es desterrar los numerosos errores y desatinos que vocabularios anteriores habían inculcado:

Digo que leí ejtos, por ver que muchos los traýan en las manos, y los acatauan como a oráculos de Apollo y hojas de la Sibylla, y entre ellos algunos Theólogos y predicadores. Di arcadas de afco en entendiendo jer tan bárbaros libros, llenos de tantas necedades y bouerías, amanzillados con tantas mentiras y vicios, tan llenos de defuarios, y de adeuinaciones burladoras y phantáfticas, tan negligentes en la orthographía, accentos, y etymología: tan inciertos en la jignificación de los vocablos, que a vna me $\int \mathrm{ma}$ dictión bueluen en más figuras y formas, que las que tuuo entre los poetas Empuja. Y finalmente vi Jus interpretaciones y declaraciones no fólo no dar en el fiel, más ni en todo el tercero. Pues no quiero aquí dezir Jus declaraciones de la Scriptura ridículas (y no quiero ponerles otro nombre peor) las fal as interpretaciones de los nombres proprios de la $\mathrm{Bi}$ blia, y el dexarje en el tintero no fólo muchos cientos, más muchos millares de vocablos. Finalmente en cada parte andan a ciegas aquellos ciegos ade ftradores y guías de ciegos, y hablan lo que no fienten. O doctores, o figlo defuenturado, o ignorancia (por no la llamar dejuergonçada arrogancia) de ejcritores, que no tienen empacho de publicar cojas jeme-

\footnotetext{
${ }^{3}$ Utilizo un ejemplar (Madrid, «Apud D. Antonium Mayoral», 1770) de una biblioteca particular.

${ }^{4}$ Véase también a este respecto el Conde de la Viñaza, Biblioteca Histórica de la Filología Castellana, III (Madrid, Manuel Tello, 1893), ed. facsimilar Madrid, Atlas, 1978, n. ${ }^{\circ}$ 1360 , pág. 938
} 


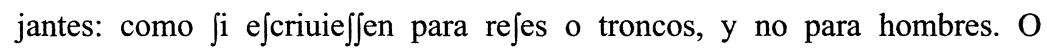
eftragados ingenios y tales para tales, los que en tales autores gajtan mal

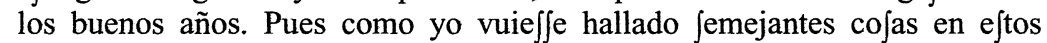
Vocabularios: parecióme que deuía dar les luego de mano, por no adquirir fciencia peor que qualquier ignorancia, acordándome de aquello que S. Hieronymo, No aprenda la niña en ju tierna edad, coja que aya de dejaprender dejpués ${ }^{5}[\ldots]$.

Estas duras palabras se refieren, aunque no lo mencione directamente, al Vocabularium ecclesiasticum de Rodrigo Fernández de Santaella, al que Diego Jiménez Arias, en su deseo de innovación, critica con una asiduidad que no pasaría desapercibida para cualquiera de sus usuarios. Muestra de ello son las más de 140 referencias negativas al Vocabularium que se hallan a lo largo de todo el Lexicon. La naturaleza de esas críticas, tal como se nos advertía en el prólogo, es muy variada:

\section{A) ATAQues POR CUESTIONES DE ORTOGRAFÍA:}

- Amphora, ræ. penultima correpta. (no Anfora, como ejcriue Rodericus de Sancta Ella). Vna vajija de barro con dos orejas o a as. Era también cierta medida de. 8. congios (que jon 48. Jextarios) llamada por otro nombre Quadrantal.

- Anus, ni.: penultima producta, qui \& Podex. La parte por donde el vientre je purga por cámara. 1. Regum. 6. Mal lo ejcriue Rodericus Sancta Ella. duplici. nn.

- Hypante, tes. Griego. Obuiatio honoris cauja. Antonius Nebrifjenjis. Llámaje anjí la fiejta de la Purificación, porque Simeón y Anna Jalieron a recebir a Chrifto. Ita opinor Jcribendum in Kalendas y no Ipopanti, o Ypopanti contra Rodericus Sancta Ella.

- Redeo, dis, diui, ditum. Tornar a algún lugar. Inde Reditio, onis, \& Reditus, tus. La buelta y tornada de lugar. Item Redeo, dis, dij. Rentar la hazienda. Inde Reditus, tus. La renta de cada vn año, o la que viene in portatis: eo quod redeat ex hæreditate. Parece que ay también razón para dezir que viene de Reddo, is. Biblia. Si viene de Reddo, efcriuirje ha dupli dd. alioquin jimplici. d, contra Rodericus Sancta Ella.

- Seminiuerbius, bij (non Semiuerbius, contra Rodericus Sancta Ella) El parlero, lenguaraz. Jembrador de palabras, hablador.

\footnotetext{
${ }^{5}$ Véase las palabras que Diego Jiménez Arias dedica al lector, traducidas al romance por el impresor Andrés de Portonariis (Salamanca, 1566). Utilizo el ejemplar conservado en la Biblioteca Nacional de Madrid con la signatura R/26700.
} 
A jsí llamaron a S. Pablo. Acta apoftolorum. 17. vbi Gręce eft Spermologus; que también Jignificat otras cojas.

B) La etimología COMO OBJeto DE CRítica:

- Aggero, ras. penultima correpta. Amontonar vna coja jobre otra [...]. No es Griego, como lo penjó Rodericus Sancta Ella. Hinc Aggeratio, onis. Aquella obra de amontonar. Et Agge ftio. onis, \& Agge tus. tus. Aquel amontonamiento, colmo, y ayuntamiento.

- A r a, ræ, foeminino. Altar o ara. No es Griego como pienja Rodericus Sancta Ella. Hara, con H. Jignificat çahurda de puercos, morada de aues. Item Ara es nombre de vna ciudad de Arabia y de vna ifla. Biblia.

- Di f colus, 1i. penultima correpta. Griego. Hombre de mala condición, crudo, rigurojo inhumano, áfpero, incomportable, malo de Jufrir. I. Petri. 2. vbi tamen Græca non habent difcolis, Jed Scolijs i. prauis fiue ajperis. Difcolus es Jubftantiuo, y efcríue Je fin h. Las etymologías que le da Rodericus Sancta Ella fon faljas.

- PræJtolor. aris. penultima producta. Ejperar lo que ha de venir Exodus. 18. las etymologías de Rodericus Sancta Ella Jon ridículas.

- Vi p e r a, ræ. penultima correpta. La bíuora. Sic dicta, porque pare biuos los biuoreznos, y no hueuos como las otras Jerpientes. Como fi la llamaran Viuípera. La etymología de Rodericus Sancta Ella. y de otros ( ( cilicet, Vipera, quaji vi pariens) es falja, como es fal $\int o$ también ju fundamento recebido de muchos doctos mal, que dizen, que los hijos matan a la madre por Jalir del vientre: cuyo contrario es cierto y experimentado [...].

\section{C) Discusión de la CANTIDAd vocálica:}

- A bi e zer. penultima producta y no la 'vltima acuta, como dize Rodericus Sancta Ella. Fue hijo de Galaad. Iojué. 17. El qual Je nombra por otro nombre Iezer, cabeça de los Iezeritas. Numeri. 26. Item vno de las fuertes y valientes de Dauid. 2. Regum. 23.

- Antiphona, næ. penultima producta y anjí el accento en ella contra el vulgo y Rodericus Sancta Ella. Porque en Griego Je efcriue con w que es luenga. Griego Interpretatur Voz recíproca, de dos coros que dizen o cantan a vezes. S. Ignatij.

- A p o log i a, æ. penultima correpta, \& accento. in eadem Jecun-

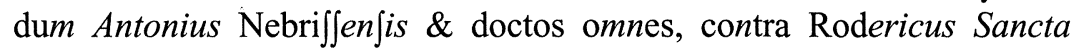


Ella. Porque los Griegos la escriuen con iota, como los otros compuejtos de Logos [...].

- Decapolis, lis, \& decapoleos. penultima correpta. Griego. Interpretatur Decem ciuitatum, Jub. regio. Región o comarca de diez ciudades. Ejtá efta región allende el Iordán, puejta ala parte del medio día de Iudea. De qua Marci. 5.7. \& Matthei. 4. No digas con Rodericus Sancta Ella en genitivo Decapoleós, 'vltima acuta. fino penultima correpta Decapóleos.

- D e c o r, or is. penultima producta. majculino. no penúltima correpta como pienja Rodericus Sancta Ella. Belleza y hermojura, o gracia con hermosura [...].

D) CRíticas SOBRE LAS INFORMACIONES GRAMATICALES:

- Actito, tas. penultima correpta. frequentatiuum, no de Ago (como dize Rodericus Sancta Ella) Jino de Acto. Y dízeje propriamente de las caujas y pleytos. vt, Actitauit caujas. S. Nicolai.

- Cancer, cri. mafculino. El cangrejo, marifco. Item vno delos 12. Jignos celeftes. Item vna enfermedad dicha çaratan, que aun que nafce en todo el cuerpo, más en los pechos de las mugeres. Es majculino Jegunda y no neutro tercera contra Rodericus Sancta Ella 2. Timotheum. 2.

- Concordo, das (no Concordor, como Rodericus Sancta Ella quiere) Concordar vna cofa con otra, Jer de vn acuerdo. Acta apoftolorum 15.

- Fornicor penultima correpta. aris. deponens verbum. Fornicar o andar en dejoneftas carnalidades y ramerías. Tranflatiue. enla. Biblia. idolatrar. Amos. 7. Es deponens verbum y no pajsiuo, contra Rodericus Sancta Ella [...].

- Plerique, pleræque. penultima producta. $\mathrm{p} l$ e r a qu e. penultima correta. Los más, o la mayor parte. No tiene jingular, como imaginó Rodericus Sancta Ella ni Je vja Plerufque. I. Prólogo Biblia. Hinc. plerumque, aduerbium. Quafi jiempre.

E) LAS SIGNIFICACIONES COMO OBJETO DE DISCUSIÓN:

- A b, Prepofición es de ablatiuo: y lo mejmo que A, o quaji lo mejmo [...]. đ Ab, diction Hebraica Jignificat dos cojas. Lo vno Paternatura, dignitate, ætate, doctrina. Lo otro vno de jus doze mejes, 
que rejponde al nuejtro Iulio: y no (como dize Rodericus Sancta Ella) a Agofto. Porque a Agofto refponde Elul. Vide Menfis.

- Brabium, Jiue etiam Brabeum, bij, \& bei. penultima producta. Griego. Interpretatur. Victoria, vel certaminis pramium. Es la joya, o palio o otro qualquier premio de la victoria, alcançada no fólo al correr (como pienja a Rodericus Sancta Ella) mas en qualquier otro dejafío y exercicio hecho jobre porfía y apuejta [...].

- Gibbus, bi. propriamente La corcoba de efpaldas o de pecho, contra Rodericus Sancta Ella. Pero algunas vezes fignificat qualquier hinchazón del cuerpo. Ejaie. 30. Gibbus, a, um. Coja corcobada. Leuítico. 21.

- Recalua ter, Jtri. mafculino. diminutivo de Caluus. El que es vn poco caluo en la frente, y no del todo: ni (como dize Rodericus Sancta Ella) caluo enel colodrillo.

- Te b e th. Hebreo. Latino. December. No Enero, como Rodericus Sancta Ella dize. Hejter. 2.

\section{F) CRÍtica DE LA INTERPRETACIÓN DE LOS TEXTOS SAGRADOS:}

- A b d e nago. Hebreo. Abednego. penultima correpta (qui \& Azarias) Nombre proprio de vno de los niños que fueron echados al fuego. Interpretatur Seruus claritatis, vel Jeruus anxius: y no (como Rodericus Sancta Ella, interpreta) Jiruiendo callo. In Danielis libro.

- C a m e r a, ræ. penultima correpta. Griega. La bóueda, y el arco que firue de Justentar el edificio. 3. Regum. 7. adonde no jignificat cámara, contra Rodericus Sancta Ella. Et. 4. Regum. 10. adonde Jegún el Hebreo ejta caja de trejquilar ouejas.

- De di co, a s. penultima correpta. Dar, aplicar, o confagrar a Dios. Biblia. Deuteronomio. 20. eftá Jegún Robertus Stephanus contra Rodericus Sancta Ella. por habitar y morar la caja nueua. Item Jecundum antiquos eft exponere. vt, Legati dedicant mandata. i. exponunt. Hinc Dedicatio, onis. La mejma penultima correpta trijýlla$b a$ tienen los otros compue $\int t o s$ de Dico.

- Lib a n s, ni. penultima correpta. Vn monte altífimo en Syria: adonde ay gran copia de encien $\int \mathrm{o}$, y altos y hermojos cedros, y otros árboles preciojos y aromáticos. En Zacharias, capitulo II. y Ezechiel. 17. Libanus fe toma por aquel templo hecho de madera del Líbano: mas no en Ejaías. capitulo. 33. contra Rodericus Sancta Ella. En el Ecclejiáftico. capitulo. 24. \&. 39. efte nombre Libanus griego que dixo el intérprete, no es nombre de árbol (como quiere Rodericus 
Sancta Ella) mas fignificat encienjo, como lo traduxo Noua Tranflatio Biblice Roberti Stphani.

El deseo de censura lleva a Jiménez Arias a introducir en la macroestructura de su diccionario términos con la ortografía que él cree correcta en el lugar que le correspondería según la ortografía empleada por Fernández de Santaella; tal es el caso de A u tor, voz que recoge en el lugar que se le asigna a A u ctor en el Vocabularium ecclesiasticum. Más aún, llega a escribir algunos términos copiando a Fernández de Santaella con el único fin de arremeter contra él, por ejemplo:

- D i m e rgo no lo ay, fino demergo. contra Rodericus Sancta Ella.

- Libitus, tus, que Rodericus Sancta Ella pone por plazer, o voluntad o antojo, no Jé fi lo ay. Pero Jé que ay Libitum. vt, Pro libito. \&, Ad libitum.

- Myrrhum, no lo ay Iudit. 10. Jino Myrtum [...]. Contra Rodericus Sancta Ella.

- S i mma, æ. Rodericus Sancta Ella dize que es la cámara, y Simmifta, el camarero. S. Sylueftri. Yo no lo hallo.

- Stir po, no lo ay, Jino Extirpo, contra Rodericus Sancta Ella.

- Volumentum, no lo ay Sapientiæ. 7. como pienja Rodericus Sancta Ella fino Inuolumentum. Y allí eftá efcrito In inuolumentis.

Algunas de las críticas son de gran dureza, tachando de ridícula la interpretación dada por Fernández de Santaella, según se puede ver en artículos como Aby $\iint u s, \int s i$, A 1 i enigena, æ, P 1 agiarius, rij o Præ $\int$ tolor, aris; otras rozan la ironía, tales son los casos de Cocytus, ti, Germanus, a, um o Semicinctium, ctij. A veces, sin embargo, el ataque no es tan directo y Jiménez Arias remite simplemente al hecho sin nombrar al autor del error que, como es obvio, no es otro que Fernández de Santaella. Basta para comprobarlo cotejar algunos artículos de ambos diccionarios ${ }^{6}$ :

- I u b a . b e . feminini generis. las crines de cauallo o de león o de otro animal bruto. chryfoftomus in decollatione fancti Johannis [...] (Santaella).

— I u bæ, a r u m. Las cernejas o crines de los animales. No Jé ji ayuba en Jingular. Chryfoftomus. in Decollatione. Baptista (Arias).

\footnotetext{
${ }^{6}$ Para el diccionario de Rodrigo Fernández de Santaella he utilizado el ejemplar de la edición sevillana de 1499 conservado en la Biblioteca Nacional de Madrid con la signatura $\mathrm{I} / 1408$.
} 
- Scabro. bronis. no fcabro. ni fcrabro. media producta. gujano que najce de carne podrida de cauallo. algunos dizen que es como abijpa \& tiene más luengos pies. es muy dañojo y enojojo [...] (Santaella).

- S c abro. No lo ay (Arias).

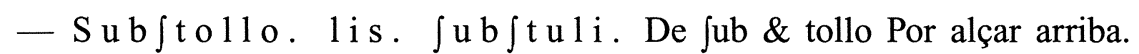
Ejaie. lviij (Santaella).

- Subftollo, no lo hay, fino Suftollo (Arias).

- Te $m$ porinus. na . nu m. penultima producta. coja de tiempo prejente. es adiectiuo diriuado de tempus. y a ajj je ha de exponer $\int \mathrm{i}$ fe falla maxime in ambro jsio enel fermón de vn confe $\iint o r$ pontífice (Santaella).

- Temporinus, a, um. No es vocablo Latino ni Griego ni Hebreo (Arias).

En total, Diego Jiménez Arias menciona a Fernández de Santaella en más de doscientas ocasiones, y no siempre para criticarlo. En la «Inftructión para entender las abreuiaturas de efte libro»" nos advierte que «Rod. Rodericus Sancta Ella, autor de vn Vocabulario ecclefiáftico. Quando aquí Je cita fin reprehenfión, es quando no jé ji es verdad lo que dize, o no»:

- Agonifticus, a, um. Dize Rodericus Sancta Ella que es el victorio $\int \mathrm{o}$, o belicofo.

- Condulco, cas. Dize Rodericus Sancta Ella que es el hazer dulce a alguna coja, o hablar cojas dulces. Ecclejiajticus $27 \& 40$.

- De int us, aduerbium. Jecundum Rodericus Sancta Ella. De parte de dentro. Lucas. II.

- Emercor, c a ris. Adquirir o ganar Jegún Rodericus Sancta Ella el qual dize que enla legenda de S. Catherina eftá pajsiue.

- In c a $\int$ e a tus, a, um. Rodericus Sancta Ella dize que S. Auguftín lo toma y vja por cofa cuajada como que jo.

- Platonia, æ. foeminino. penultima correpta. Dize Rodericus Sancta Ella jer lugar donde echaron los cuerpos de los apóftoles. S. Damaji.

- Sellarius, rij. Dize Rodericus Sancta Ella que es nombre proprio. S. Anajtajij.

- The atru m, tri . penultima producta. Griego. Vn edificio de medio círculo, hecho para ver las reprejentaciones y jpectáculo [...]. Dize Rodericus Sancta Ella que in vita de S. Agnetis je poner por burdel. 
- Thermate s, t is. penultima producta. vel Thermas. atis. Dize Rodericus Sancta Ella que es la veftidura caliente que arropa y abriga S. Hilarionis.

- Vociferor. penultima correpta. aris. deponens verbum. Gritar y dar bozes. Vociferatio onis. La obra de dar tales bozes. Hieronymo. II. Dize Rodericus Sancta Ella que muchas vezes Je halla Vocifero, as. penultima correpta. neutrum. S. Proceji \& Martiniani.

Esa gran cantidad de citas o alusiones a Fernández de Santaella no deja de ser sorprendente. ¿Cuál puede ser el motivo de que Jiménez Arias recurra en tantas ocasiones a un autor al que - como hemos visto - critica tan duramente? ¿Por qué cita a Fernández de Santaella si pretende demostrar en todo momento que su diccionario no tiene nada que ver con el de éste? ${ }^{7}$

La respuesta a esas preguntas se halla en el objetivo mismo con que nace el Lexicon ecclesiasticum. Jiménez Arias pretende elaborar un diccionario normativo, capaz de fijar la ortografía, la etimología, el significado, etc., de las palabras latinas y de terminar con los errores cometidos por obras lexicográficas anteriores. Con este fin, toma como punto de partida los diccionarios latinos más importantes y de mayor difusión en la época; de ahí que no pueda dejar al margen, a pesar de los muchos errores que ve en él, el Vocabularium ecclesiasticum de Fernández Santaella ${ }^{8}$, pero parece que

\footnotetext{
7 «Tratando conmigo algunos amigos jobre limpiar eftos Vocabularios, algunos me importunauan que yo tomafe efte trabajofífimo cargo. Otros que tenían cuenta con mi honra, me aconjejauan lo contrario: porque la gloria que a mi $\int \mathrm{e}$ me deuie $\iint \mathrm{e}$ por lo bien añadido, no boluie $\iint e$ a los primeros autores delos libros anjí acrecentados, como ha acontecido a los doctífimos acrecentadores de Calepino. Antes me juadían, que fin yr arrimado a nadie, hizie $\iint e$ de mi cofecha vn nueuo Vocabulario [...]. Mouido pues yo con femejantes razones, y con los ruegos de los amigos, difpuje me a hazer vn nueuo Vocabulario ecclejiáftico. Pero dejpués de començado, parecióme tan difficulto 0 el negocio, que cierto amaynando las velas, dexara mi nauegación començada, fi los ruegos de los amigos, no boluieran a acabar conmigo que acabajfe lo començado. Recibe pues amigo Lector el nueuo Dictionario ecclefiático (que Je llama en Griego Lexicon ecclejiafticum) hecho de mi cabeça y induftria con trabajos enteros diez años, tan grandes, que apenas los podrá nadie cree: el qual te doy agora, por cofa que te ha de aplazer y contentar con más que con la nouedad [...]» (prólogo romance de la edición salmantina de 1566. Véase la nota número 5). En términos semejantes, o más precisos si caben, se expresa el impresor Andrés de Protonariis en las palabras que dirige al lector: «Recibe pues benignamente efta obra nueua que podrá aprouechar a doctos y indoctos: a monjas, y otras perjonas de poco, o ningún latín, anfí en accentos como en jignificaciones de dictiones. Y no digas que ejte es el Vocabulario antiguo de Rod. de Sancta Ella anadido: pues tiene muy mucho menos que ver con effe (como lo verás cotejándolos) que con Calepino y el Vocabulario de Lebrixa: pues que de eftos dos tomó muchas cofas, y ninguna de aquél [...]».

${ }^{8}$ El Vocabularium ecclesiasticum de Rodrigo Fernández de Santaella, antes de la edición corregida y aumentada por Diego Jiménez Arias en 1566, había sido ya impreso, al menos, en treinta ocasiones. Véase Joaquín Hazañas y la Rúa, op. cit. (1990), pág. 39.
} 
sólo lo cita «jin reprehenfión» cuando encuentra en él informaciones que no se hallan en los repertorios de Nebrija ${ }^{9}$ o Calepino ${ }^{10}$, los autores más citados en el Lexicon ecclesiasticum después de Fernández de Santaella ${ }^{11}$.

Como hemos visto, en la «Inftructión para entender las abreuiaturas de este libro» Diego Jiménez Arias afirma que cuando cita a Rodrigo Fernández de Santaella sin hacer ningún tipo de crítica o corrección es porque no sabe si lo que dice el autor del Vocabularium ecclesiasticum es verdad o no. $\mathrm{Su}$ intención es poner una vez más en entredicho la obra de Santaella, pero el verdadero motivo de que en numerosas ocasiones no pueda corregir el texto del Vocabularium ecclesiasticum se debe a que en España no se conocía otro diccionario destinado a la correcta interpretación de los Textos Sagrados, a facilitar su comprensión a los eclesiásticos con escasos conocimientos de latín. Aquí precisamente reside el gran éxito del que gozó durante siglos el Vocabularium ecclesiasticum y por qué, a pesar de los reproches de Jiménez Arias, era alabado y consultado por numerosos teólogos.

\footnotetext{
${ }^{9}$ De las obras de Nebrija, Diego Jiménez Arias sólo hace mención directa al Lexicon iuris civilis (Arrha, rrhce \& Arrhabo; Daphne, nes; Vicarius, a, $u m$, etc.) y a la Tertia quinquiagena (A rceutinus, a, um; Michol; Pa cha, $a, P h y t o n, o n i s$, etc.). No obstante, parece obvio que el autor del Lexicon debió de tener presente, dado su enorme prestigio, los diccionarios de Nebrija, al menos el Diccionario latino-español, pues el Lexicon ecclesiasticum es un diccionario monodireccional y sólo sirve para traducir del latín al español. La influencia del Diccionario latino-español de Antonio Nebrija se deja entrever con claridad, por ejemplo, en Adoleo, les. 1 u i \& leui, adolitum. \& adultum; Aeuum. ui; Amarico, cas; Atrium, trij; Bucca, ccæ, Capparis \& Cappar. ris; Dama, mæ: Lamia, æ, Jiue Lamiæ, arum, Mane o Sanguino, nas .

${ }^{10}$ El Dictionario de Calepino se publicó por primera vez en 1502. La obra tuvo un verdadero éxito, y a ella recurre Jiménez Arias, no sólo para fijar el significado de las entradas latinas, sino también como fuente de ejemplos, pues con frecuencia remite al lector del Lexicon ecclesiasticum al Calepino con frases como éstas: «Los ejemplos de esto mira los en Calepino», "Horum exempla. vide in calepino» (véanse entradas como A b, A d, B o n u s, $\mathrm{E}$ o, etc.). Y es que los ejemplos, tal como lo demuestra la asidua presencia de éstos en los artículos del Lexicon y el reproche que dirige a Nebrija en el prólogo romance de la edición de 1566 por la escasez de ejemplificación de sus diccionarios, constituyen una parte importante del proyecto lexicográfico de Jiménez Arias.

11 Véase Antonia M. ${ }^{\mathrm{a}}$ Medina Guerra, Los diccionarios bilingües con el latín y el español (1490-1619), edición en microfichas, Málaga, Servicio de Publicaciones de la Universidad, 1994, n. $^{\circ} 97$.
} 